\title{
3D Reproduction of Oil Painting Based on UV Ink-Jet Technology
}

\author{
Yansong Peng ${ }^{1, a}$, Chen Chen ${ }^{2, b^{*}}$, Liuxi He ${ }^{2, c}$, Haozhi Chen ${ }^{3}$ \\ ${ }^{1}$ The Affiliated High School of South China Normal University, Guangzhou, China \\ ${ }^{2}$ State Key Laboratory of Pulp and Paper Engineering, South China University of Technology, \\ Guangzhou, China \\ ${ }^{3}$ Faculty of Mathematics, South China University of Technology, Guangzhou, China \\ apengys.harry@outlook.com, b977092@qq.com (Corresponding author), cliuxi_he@sina.com
}

\begin{abstract}
Keywords: Oil Painting; UV ink; 3D Printing; Stratification Processing; 3D Reproduction
Abstract. The goal of this experiment is to use UV ink as the modeling material to reproduce oil paintings with colorful three-dimensional features. The scanned picture of oil painting stored in bitmap format was sent into a software program that provided stratification processing. The layers were printed on paper or canvas using 3D print technology in the UV-LED ink-jet printer, with a sequence of positive and reverse outputs, and two output methods consisting of a combination of multilayer white ink with a layer of colored ink, and a combination of a layer of colored ink with multilayer transparent ink. The results indicated that replicating oil paintings with fine three-dimensional details and vivid color effects could be realized when the layers after stratification were printed out using a reverse sequence of multilayer white ink and colored ink. Therefore, UV ink-jet technology can successfully realize the reproduction of oil paintings with colorful three-dimensional features.
\end{abstract}

\section{Introduction}

Living standards and consumption are constantly improving, it can be seen that demand for oil paintings and other forms of art also increase. World-famous oil paintings are difficult to obtain for average consumer. To produce exact replicas of oil paintings, artists need high precision instruments and equipment. Currently, there are two methods to obtain high quality reproductions of oil paintings: manual reproduction and digital printing. Manual reproduction using oil paint pigment can achieve a real sense of texture, but the process is time-consuming. Its producing cycle is too long, and its quality is limited in a great degree. Thus, manual reproduction cannot keep up with the market demand. Ink-jet printing is the other method of reproducing artwork. With the use of new high precision technology that has improved speed, we still cannot reproduce the three-dimensional texture we see on the surface of oil paintings.

The appearance of 3D printing technology has provided a method for the reproduction of oil paintings. However, 3D printing still has limitations, as the color is not rich enough. In recent years, researchers are focusing on light curing the materials. Domestically, the three-dimensional printer nozzle, the stereo lithography apparatus system and the corresponding molding material were independently developed at Xi'an Jiaotong University, and the forming precision was within $0.2 \mathrm{~mm}$ [1]. H.M. Wang successfully printed a three-dimensional topographic map based on UV ink-jet printer [2, 3]. Abroad, 3D Systems developed a light-cured 3D printer using photosensitive resin as the molding material in 2004 [4]. UV ink is a typical light curing material, containing light initiator, pre-polymer and monomer, which can incur a polymerization reaction and form glossy, colorful ink layers in a short time under the irradiation of a UV light. With no volatile solvents, these characteristics and properties make UV ink particularly suitable for 3D printing molding material [5, 6]. This paper presents a new method for the 3D reproduction of oil paintings based on open software and a UV-LED ink jet printer. The method does not require high precision equipment to support, but can reproduce fine replicates of three dimensional oil paintings. 


\section{Experiment}

Equipments. Mimaki UJF-3042 UV ink-jet printer; Mimaki RasterLinkPro5 IP; Adobe Photoshop CS6; Adobe Illustrator CS6.

Materials. MimakiLH-100 CMYK; MimakiLH-100W; MimakiLH-100Cl; Coated paper; Canvas.

Acquisition of oil painting. Having searched the Internet for oil paintings with the appropriate content, image size and file size, we chose three, as shown in Fig. 1, Fig. 2 and Fig. 3.

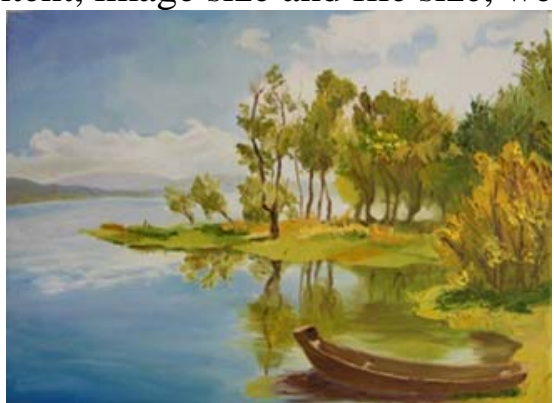

Fig. 1. Scenery

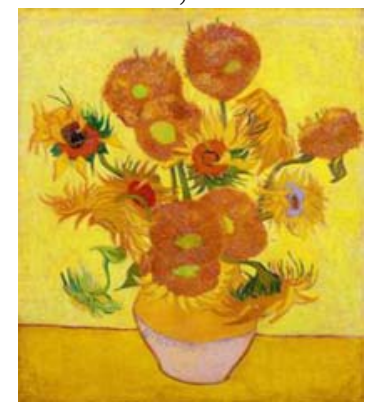

Fig. 2. Sunflower

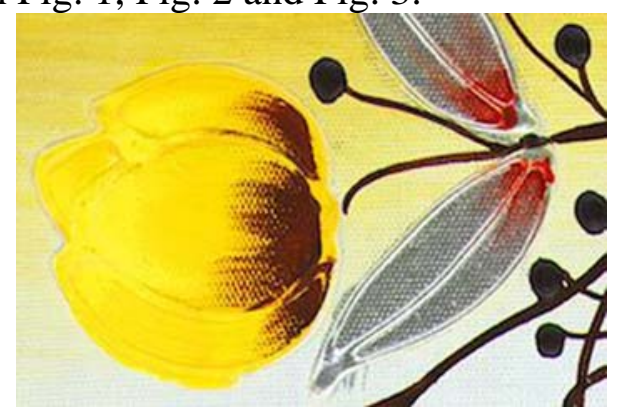

Fig. 3. Tulip

Stratification processing of oil painting. Using Adobe Photoshop CS6 software, we selected and built areas to stratify the picture, according to the high and low elevation on the oil painting. The areas were respectively converted to stratification layers stored in bitmap format, as shown in Fig. 4, which was stratified as an example from Fig. 2. The sequence numbers were labeled from the bottom to the top in accordance with the elevation of the surface of the oil painting.

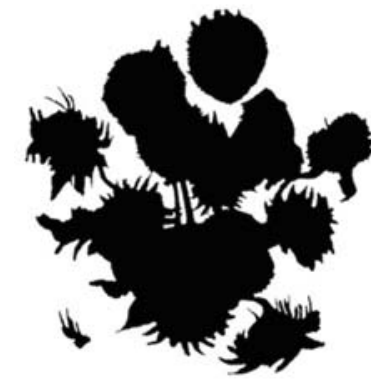

1

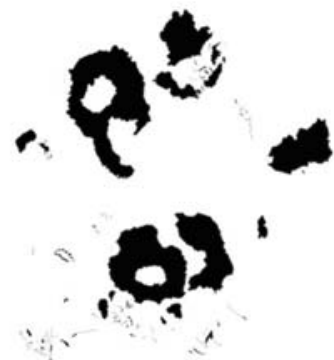

2

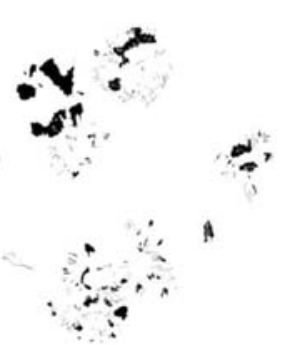

3

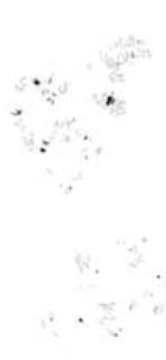

4

Fig. 4. Stratification processing

Vector of stratification files. In the software of Adobe Illustrator CS6, we portrayed and extended every stratification layer and saved it in a vector format, in order to use the white ink (or transparent ink) replacement function in the UV ink-jet printer.

3D Reproduction of Oil Painting. Four alternate methods were examined:

a. Import the stratification layers into UV ink-jet printer in positive sequence of sequence numbers, use white ink replacement function to print every layer, and finally use colored ink to print a colorful picture of oil painting.

b. Import the stratification layers into UV ink-jet printer in positive sequence of sequence numbers, use colored ink to print a colorful picture of oil painting, and finally use transparent ink replacement function to print the stratification layers.

c. Import the stratification layers into UV ink-jet printer in reverse sequence of sequence numbers, use white ink replacement function to print every layer, and finally use colored ink to print a colorful picture of oil painting.

d. Import the stratification layers into UV ink-jet printer in reverse sequence of sequence numbers, use colored ink to print a colorful picture of oil painting, and finally use transparent ink replacement function to print the stratification layers. 


\section{Results and Discussions}

Comparison and Analysis of Substrate. Fig. 5 showed the scenery (Fig. 1) printed on coated paper, and Fig. 6 showed the scenery printed on canvas. By comparison, we could find that the oil painting printed on coated paper had bright colors and high brightness, due to the high smoothness, gloss, whiteness and light reflectivity of the paper. But the uneven texture on the surface of scanned original canvas was also reproduced, so that the replica had a strong granular sensation and a weak sense of reality. In addition, a certain thickness of ink accumulated on paper surface would shrink and lead to the deformation of the paper, which could eventually reduce the quality of replica. The oil painting printed on canvas had high color saturation, natural color transition, and obvious three-demensional effect, because of the canvas with high roughness and low reflectivity, although the color gradation was relatively dark. Moreover, the soft canvas could buffer the shrinkage of ink making the replica deform only slightly. In summary, canvas is a more suitable substrate for reproducing the oil painting.

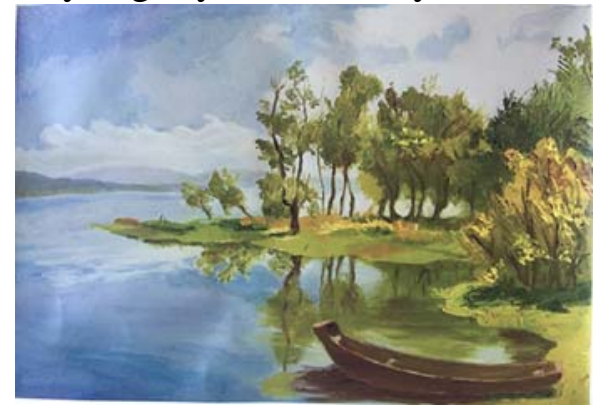

Fig. 5. Scenery printed on coated paper

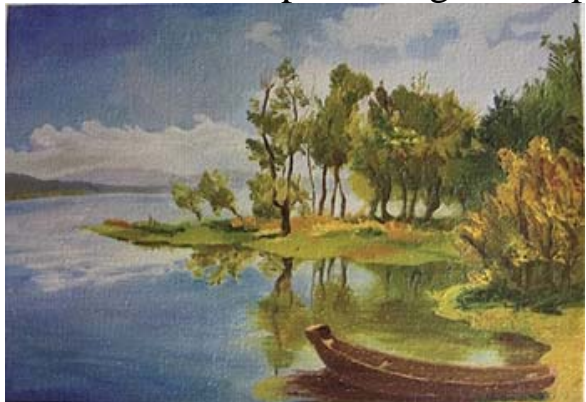

Fig. 6. Scenery printed on canvas

Comparison and Analysis of Ink. Color cast and low brightness would appear when multilayer ink accumulated, mainly caused of the optical difference of cyan, magenta and yellow ink [7]. Therefore, colored ink could only be printed once on substrates. There were two ways to achieve the 3D reproduction of oil painting on the basis of previous studies. The first method was to print multilayer white ink to achieve the height of oil painting, and then to print a layer of colored ink on the multilayer white ink to achieve the color of the oil painting. The second method was to print a layer of colored ink on canvas to achieve the color of the oil painting, and then to print multilayer transparent ink on the layer of colored ink to achieve the height of the oil painting. A part of Fig. 2 was selected to print in this experiment. Fig. 7 showed the result through the first method, and Fig. 8 showed the result through the second method.

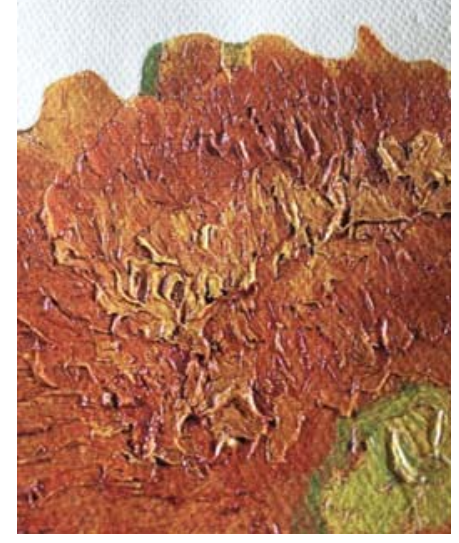

Fig. 7. Sunflower printed on canvas by white ink

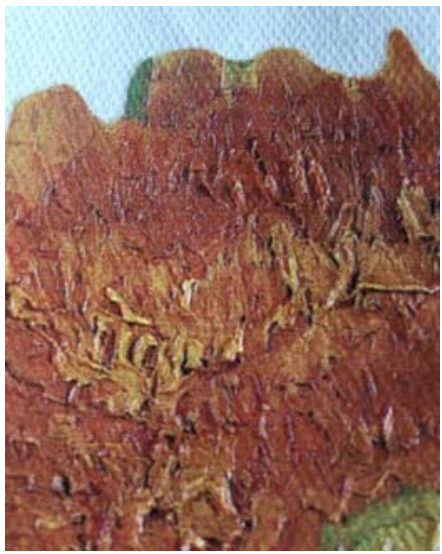

Fig. 8. Sunflower printed on canvas by transparent ink

Through comparison, it was found that the accumulation of transparent ink affected the light transmission, resulting in a dim color and a low color saturation of colored ink at the bottom of transparent ink, and a weak three-dimensional sense. While a high degree of color saturation and brightness, and a strong three-dimensional sense were obtained by the accumulation of multilayer 
white ink and a layer of colored ink on top. In addition, white ink could effectively improve the color cast [7].

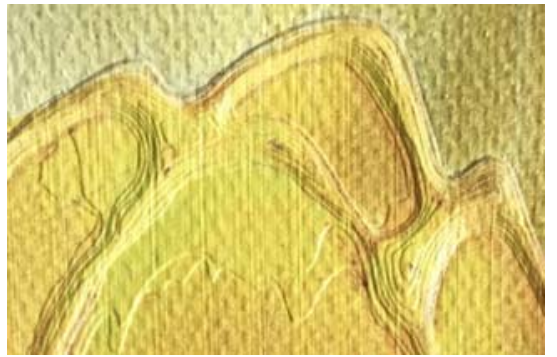

Fig. 9. Tulip printed on canvas by white ink in positive sequence of stratification layers

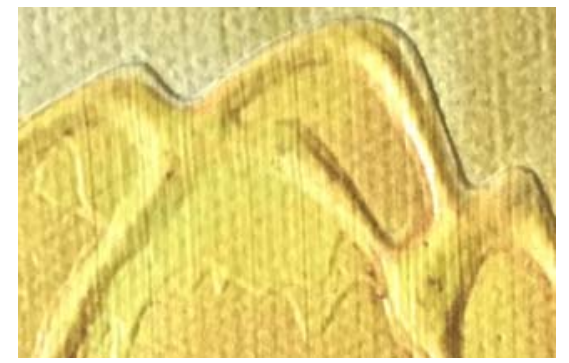

Fig. 10. Tulip printed on canvas by white ink in reverse sequence of stratification layers

Comparison and Analysis of Printing sequence. The general sequence of 3D printing was from the bottom to the top of the model. The reverse sequence was used to print the oil painting in this experiment. Fig. 9 showed the result printed in positive sequence of stratification layers, and Fig. 10 showed the result printed in reverse sequence. The results obvious showed that the elevation printed in reverse sequence had a continuous transition effect, which had greatly improved the terrace phenomenon generated from printing in positive sequence. That was because the upper layer of ink would accumulate on the lower layer of ink. When the stratification layers were printed in reverse sequence, a smooth and continuous curved surface would form, since the area of the upper layer was larger than that of the lower layer.

\section{Conclusion}

Based on our work, the best method for the reproduction of oil paintings proposed that the stratification layers stratified and numbered from oil paintings would be imported into the UV ink-jet printer and printed in reverse sequence of sequence numbers with multilayer white ink achieving the height of the oil painting and then a layer of colored ink printed on the multilayer white ink achieving the color of the oil painting. Fig. 11, Fig. 12, and Fig. 13 show three reproductions from Fig. 1, Fig. 2, and Fig. 3. The advantages of this method were: (a) it could reduce the technical difficulty of reproducing oil paintings, the average person could reproduce it just with open software and UV ink jet printers; (b) it compensated for the deficiency of common digital printing in realizing the reproduction of the surface texture and elevation; (c) it provided good technical ideas and references for 3D color printing.

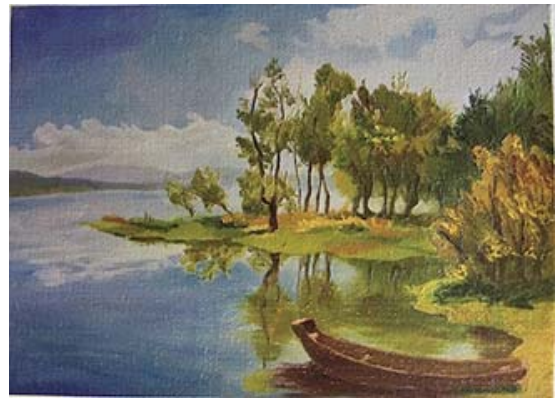

Fig. 11. Scenery replica

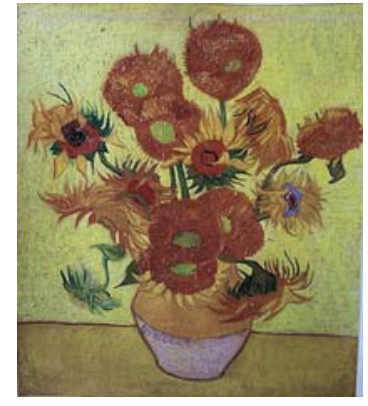

Fig. 12. Sunflower replica

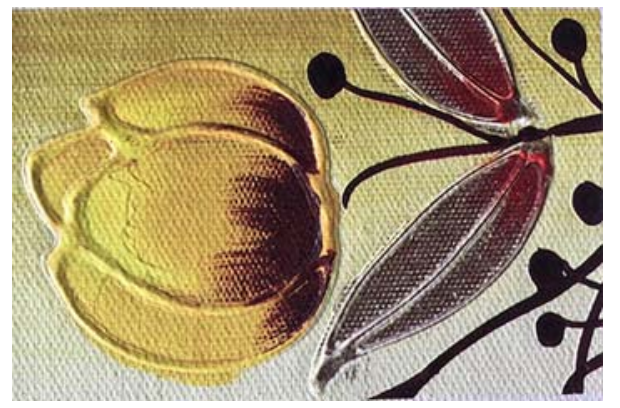

Fig. 13. Tulip replica

Of course, there still exist many problems in the application of UV ink-jet technology to print oil paintings, such as: manual stratification reduces the accuracy and reliability of the surface quality of the oil painting, ink penetration leads to some errors in reproducing the height, and high precision details are difficult to reproduce. With the development of 3D printing technology and 3D scanning technology, this method can play a role in the promotion and protection of the precious oil paintings, and possesses a high market value and market demand at the same time. 


\section{Acknowledgments}

This work is supported by the Guangdong Province Science and Technology Project (grant no.2013B010401007).

\section{References}

[1] Information on http://news.yjrb.com.cn/news/kj/665386.shtml

[2] Huanmei Wang, Guangxue Chen: Applied Mechanics and Materials Vol. 469 (2014), p. 309-312

[3] Huanmei Wang, Guangxue Chen: Packaging Journal Vol. 6 (2014), p. 48-52

[4] H. Lipson, M. Kurman: The new world of 3D printing (John Wiley \& Sons, United States 2013).

[5] Xuanwu Han: Packaging Engineering Vol. 30 (2009), p. 48-51

[6] Haisheng Chen: Packaging Engineering Vol. 33 (2012), p. 97-100

[7] Liuxi He, Guangxue Chen: Packaging Engineering Vol. 36 (2015), p. 134-138 